\title{
Multi-scale mathematical modelling of tumour growth and microenvironments in anti-angiogenic therapy
}

\author{
Yan Cai ${ }^{1,2^{*}}$, Jie Zhang ${ }^{3}$ and Zhiyong $\mathrm{Li}^{1,2}$
}

*Correspondence:
yancai@seu.edu.cn
2 School of Biological
Sciences and Medical
Engineering, Southeast
University, 2 Sipailou,
Nanjing 200018, China
Full list of author information
is available at the end of the
article

${ }^{*}$ Correspondence:

yancai@seu.edu.cn

Sciences and Medical

Engineering, Southeast

Unersity, 2 sipailou, article

\begin{abstract}
Background: Angiogenesis, a process of generation of new blood vessels from the pre-existing vasculature, has been demonstrated to be a basic prerequisite for sustainable growth and proliferation of tumour. Anti-angiogenic treatments show normalization of tumour vasculature and microenvironment at least transiently in both preclinical and clinical settings.
\end{abstract}

Methods: In this study, we proposed a multi-scale mathematical model to simulate the dynamic changes of tumour microvasculature and microenvironment in response to anti-angiogenic drug endostatin (ES). We incorporated tumour growth, angiogenesis and vessel remodelling at tissue level, by coupling tumour cell phenotypes and endothelial cell behaviour in response to local chemical and haemodynamical microenvironment.

Results: Computational simulation results showed the tumour morphology and growth curves in general tumour progression and following different anti-angiogenic drug strategies. Furthermore, different anti-angiogenic drug strategies were designed to test the influence of ES on tumour growth and morphology. The largest reduction of tumour size was found when ES is injected at simulation time 100, which was concomitant with the emergence of angiogenesis phase.

Conclusion: The proposed model not only can predict detailed information of chemicals distribution and vessel remodelling, but also has the potential to specific antiangiogenic drugs by modifying certain functional modules.

Keywords: Multi-scale mathematical model, Numerical simulation, Solid tumour growth and angiogenesis, Tumour microenvironment, Anti-angiogenic therapy

\section{Background}

Angiogenesis is a physiological process of generation of new blood vessels required for normal body functioning, such as wound healing, endometrial growth during the menstrual cycle, tissue grafting, inflammation, and hypoxia. However, clinically and pathologically it has been demonstrated as a basic prerequisite for sustainable growth, proliferation and metastatic spread of solid tumours [1]. Cascade of events activated by several pro-angiogenic factors produced by hypoxic tumour cells happens during tumour angiogenesis including dissolution of vascular basal membrane, increased vascular permeability and degradation of extracellular matrix (ECM) resulting in endothelial 
cell (EC) migration, invasion, proliferation and tube formation [2]. The growth of new blood vessels requires the concerted action of activators and inhibitors of angiogenesis. Chemicals that can activate angiogenesis include vascular endothelial growth factor (VEGF), matrix metalloproteinases (MMPs), placenta growth factor (PlGF), fibroblast growth factor (FGF) and hepatocyte growth factor (HGF) [3]. Endogenous inhibitors of angiogenesis include angiostatin (AS) and endostatin (ES) [4]. The imbalance between angiogenic growth factors and inhibitors is believed to be significantly important in the development of the tumour vasculature [5].

Given the prominent role of angiogenesis in cancer, many cancer therapies aim to block angiogenesis and thereby inhibit tumour growth. These approaches undertaken towards that goal include inhibition of growth factors required for ECs proliferation and survival, such as VEGF [6]; inhibition of MMPs [7]; or direct inhibition of EC migration [8]. In addition, vascular disrupting agents (VDA) have been shown to inhibit tumour growth through induction of vascular collapse [9]. This research was transformed into therapeutic modality which led to the development of bevacizumab (Avastin), the first VEGF targeted anti-angiogenesis cancer therapy, and approved by the FDA in 2004 [10].

While the anti-angiogenic therapy have been approved for cancer treatment, it appears that the clinical application of anti-angiogenic therapy is more complex than originally thought. Since the anti-angiogenic drugs target tumour vasculatures without tumour cells, resistance to anti-angiogenic therapy caused by the pathological microenvironment in tumour is a prominent issue which may explains the variable results observed in the experiment and clinic using this approach [11]. Unlike normal blood vessels, tumour vasculature has abnormal structure and function [12]. The microvessels inside the tumour are leaky, which causes the high interstitial fluid pressure (IFP) in tumours. The blood perfusion in the abnormal microvasculature is heterogeneous and often compromised [13]. In addition, host-tumour interactions regulate the dynamic balance of angiogenic growth factors and inhibitors and result in the pathophysiological characteristics of the tumour [14]. Therefore, it is important to understand the mechanisms of interactions between tumour cells and microenvironment in response to anti-angiogenic therapy.

Mathematical modelling and numerical simulation offer powerful tools with which to study complex biological processes, such as tumour growth and angiogenesis. Billy et al. [15] developed a model of tumour growth that includes inhibitors (ES and Ang2) and promoters (VEGF and Ang1) of angiogenesis. Sleeman and coworkers' [16] recent study proposed a model that combined angiogenesis and haemodynamic simulations in metastatic tumours. Their model predicted that treatment with angiostatin affected tumour vessels through the process called 'vessel normalization'. Jain and coworkers developed a model to predict changes in tumour microenvironment including interstitial fluid pressure after vessel normalization treatment [17, 18]. Alarcon et al. [19] proposed a model of VEGFR association with VEGF and internalization to investigate how these processes influence the response to anti-angiogenic therapy. Using numerical algorithms, such as partial differential equations, agent-based modelling, and continuous-discrete hybrid modelling, computational modelling and numerical simulation provide platforms to study the complexity of tumour growth and angiogenesis process and the therapeutic strategies of anti-angiogenesis in cancer. 
Based on the continuous-discrete hybrid model proposed by Chaplain's group for modelling angiogenesis [20], we started our work by investigating the dynamic interactions between local haemodynamics and angiogenesis in a 2D model [21] and then developed it to a 3D model [22]. The inclusion of different tumour cell phenotypes into the angiogenesis simulation forms our second generation of model in which the tumour growth, vessel remodelling and blood perfusion were incorporated [23]. The present study is a further development in which a multi-scale 3D model was established aiming to simulate the dynamic changes of tumour microvasculature and microenvironment in response to anti-angiogenic therapy. The model focused on the anti-angiogenic drug endostatin (ES), which can inhibit endothelial cell (EC) proliferation, migration, invasion and tube formation. We envision that the proposed model will serve as a simulation framework for studying tumour growth and the changes of tumour microenvironment in response to anti-angiogenic therapy.

\section{Mathematical model and methods}

Multi-scale 3D tumour modelling is utilized to detail the mechanisms of tumour growth and angiogenesis. We have organized our model into three levels: tissue, intratumoral and cellular layers. The overall structure of this model is summarized in Fig. 1. In the tissue layer, we deal with the morphology of solid tumour and the structural properties of the vascular network. Four different phenotypes of tumour cells with corresponding metabolic characteristics are introduced in "Tumour growth" section. Modelling of tumour angiogenesis due to endothelial cell proliferation and migration is presented in "Angiogenesis and vessel remodelling" section. In addition, vessel remodelling in response to varied microenvironment is also considered in "Angiogenesis and vessel remodelling" section. The tissue level and the cellular level are coupled by the changes of microenvironment in the intratumoral level, including chemicals concentration flied ("Chemical microenvironment" section) and haemodynamic information ("Haemodynamical microenvironment" section). In "Anti-angiogenic therapy" section, we will develop the multi-scale model with anti-angiogenic drug delivery to investigate the influence of anti-angiogenic therapy on the tumour growth. Initial conditions and simulation algorithm are given in "Simulation setup" section.

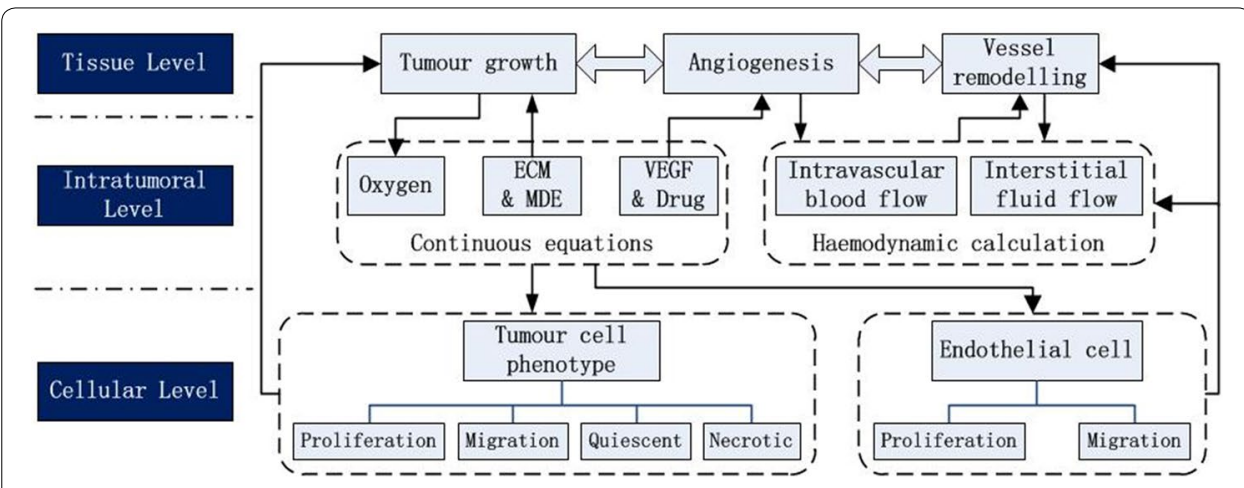

Fig. 1 Structure of multi-scale modelling system 


\section{Tumour growth}

The probabilistic hybrid model for tumour cell growth is based on the previous work [23]. We assumed four different phenotypes of tumour cells: the proliferative cells (PC), the quiescent cells $(\mathrm{QC})$, the necrotic cells $(\mathrm{NC})$ and the migrating cells (MC). Two thresholds of oxygen concentration for cell proliferation $\left(\theta_{\text {prol }}\right)$ and cell survival $\left(\theta_{\text {surv }}\right)$ are introduced to describe the effects of oxygen field on the tumour cell actions. Each phenotype of tumour cell has a different coefficient of oxygen consumption rate and the production rate of VEGF and MDEs (Table 1) [24].

\section{Proliferative cells (PC)}

If there is enough oxygen $\left(C_{o}^{e x} \geq \theta_{\text {prol }}\right)$ and space is available, a tumour cell will proliferate into two daughter cells with a probability, defined as $\mathrm{T}_{\mathrm{age}} / \mathrm{T}_{\mathrm{TC}} \cdot \mathrm{T}_{\text {age }}$ is the tumour cell age, ranging from 1 to $\mathrm{T}_{\mathrm{TC}}$ and with an incremental 1 in each simulation time step. $\mathrm{T}_{\mathrm{TC}}$ is the tumour cell proliferation time (set to be $9 \mathrm{~h}$, equal to six time steps). One of the two daughter cells will replace the parent cell and the other cell will move to a neighbouring element.

\section{Quiescent cells (QC)}

When a tumour cell satisfies the survival condition but there is no neighbouring space for it to proliferate, it will go quiescent. When the neighbouring space of one quiescent cell has been released, the quiescent cell will turn back into a proliferating cell if the local oxygen supply is sufficient.

\section{Necrotic cells (NC)}

When the local oxygen concentration at a tumour site is less than the cell survival threshold $\theta_{\text {surv }}$, the tumour cell is marked as a necrotic cell and will not be revisited at the next time step. A necrotic cell has a probability of $20 \%$ to disappear and release the space for a tumour cell or an endothelial cell if it stays necrotic for more than $45 \mathrm{~h}$ (30 time steps).

\section{Migrating cells (MC)}

When local oxygen level is higher than $\theta_{\text {surv }}$ but lower than $\theta_{\text {prol }}$ and a space is available, a proliferating cell has a probability (50\%) to become a migrating cell (MC), and will migrate to a neighbouring space which has the highest oxygen concentration in the neighbouring elements. It was also assumed that the migrating cells adjacent to the preexisting vessel wall have higher probability of moving in the longitudinal direction (vessel axial direction) than the radial direction. The migration speeds of the two directions

Table 1 Parameters of different phenotypes of tumour cells

\begin{tabular}{llll}
\hline Phenotypes & MDE production & VEGF production & Oxygen consumption \\
\hline Migrating cells (M) & $2 \mu_{\mathrm{T}}$ & $x \times 4$ & $2 \gamma$ \\
Proliferating cells (P) & $\mu_{\mathrm{T}}$ & $x$ & $\gamma$ \\
Quiescent cells (Q) & $\mu_{\mathrm{T}} / 5$ & $x \times 2$ & $\gamma / 2$ \\
Necrotic cells (N) & $\mu_{\mathrm{T}} / 10$ & $x \times 4$ & $\gamma / 4$ \\
\hline
\end{tabular}


are the same. After a migrating cell completes its movement, the space it originally occupied will be released for other cells.

\section{Angiogenesis and vessel remodelling}

A 3D hybrid discrete-continuum angiogenesis model was adopted to investigate the EC migration and proliferation through random motility, chemotaxis in response to VEGF distributions and haptotaxis in response to the local ECM density [20]. Endothelial sprouting is only allowed in immature vessels, and the endothelial cell distribution was updated based on the equation

$$
\frac{\partial \mathrm{e}}{\partial \mathrm{t}}=\mathrm{D}_{\mathrm{e}} \nabla^{2} \mathrm{e}-\nabla \cdot\left(\frac{\phi_{\mathrm{c}}}{1+\sigma \mathrm{C}_{\mathrm{v}}} \mathrm{e} \nabla \mathrm{C}_{\mathrm{v}}+\phi_{\mathrm{h}} \mathrm{e} \nabla \mathrm{C}_{\mathrm{f}}\right)
$$

where e is the EC density. $\mathrm{D}_{\mathrm{e}}, \phi_{\mathrm{c}}, \phi_{\mathrm{h}}$ are EC diffusion, chemotaxis and haptotaxis coefficients, respectively. ECs are allowed to move along the six directions in a 3D space.

In our model, we consider vessel dilation as the first sign of a pre-existing vessel becoming an immature vessel. A vessel segment inside the tumour has a VEGF concentration larger than a threshold $\theta_{\mathrm{VEGF}}$ will increase its radius $\mathrm{R}$ with the rate of $0.4 \mu \mathrm{m} \mathrm{h}^{-1}$ which will stop when the vessel radius reaches the maximum value of $R_{\max }=10 \mu \mathrm{m}$ [25]. At the same time, the permeability of the vessel wall $\mathrm{L}_{\mathrm{p}}$ is increasing in a dilation vessel, and satisfies

$$
L_{p}= \begin{cases}L_{P}^{T}\left(1-\frac{R \max -R}{R_{\max }}\right), & \text { immature vessel } \\ L_{P}^{N}, & \text { mature vessel }\end{cases}
$$

where $L_{p}^{N}$ is the initial value of $L_{p}$ referred to the vessel permeability value in the normal tissue; $\mathrm{L}_{\mathrm{p}}^{\mathrm{T}}$ is the maximum value of $\mathrm{L}_{\mathrm{p}}$ according to the experiments of vessel permeability value in a tumour microvessel.

For a pre-existing vessel, once vessel dilation occurs, the vessel segment is treated as an immature vessel with increased $\mathrm{L}_{\mathrm{p}}$. In the simulation, vessel wall compliance is defined by the radius changing under the influence of intravascular and interstitial pressures and collapse pressure based on the empirical equation of Netti et al. [26].

$$
R= \begin{cases}R_{0}\left(\frac{P_{v}-P_{i}+P_{c}}{E}\right)^{b}, & \text { immature vessel } \\ R_{0}, & \text { mature vessel }\end{cases}
$$

where $\mathrm{R}_{0}$ is the origin radius of the capillary; $\mathrm{P}_{c}$ is the collapse pressure; $\mathrm{b}$ is the compliance exponent; $\mathrm{E}$ is the compliance coefficient. The intravascular and interstitial pressures are calculated by fully coupled haemodynamic simulation, which will be detailed in "Haemodynamical microenvironment" section.

Based on the above equations, when the vessel segment becomes immature, $L_{p}$ will increase which causes higher $\mathrm{P}_{\mathrm{i}}$, and consequently vessel will be compressed. A compressed vessel, on the other hand will induce a higher flow resistance, lower flow which will then decrease the wall shear stress (WSS) level for the vessel. Vessel collapse will occur by either a significant reduced R or WSS criteria [24]. 


\section{Chemical microenvironment}

The tumour cell and endothelial cell behaviours are coupled by the changes of the chemicals in the extra-cellular matrix (ECM), such as oxygen, VEGF and matrix degradation enzymes (MDEs) [24]. The transport of these chemicals (oxygen, VEGF and MDEs) are modelled by quasi-steady reaction-diffusion equations. The ECM is treated as a continuous substance and can be degraded by MDEs, while the MDEs are governed by diffusion, produced by TCs and ECs, and the decay of itself. The equations describing the interactions of TCs and ECs with ECM, MDE and VEGF are

$$
\begin{aligned}
& \frac{\partial C_{f}}{\partial t}=-\delta C_{m} C_{f} \\
& \frac{\partial C_{m}}{\partial t}=D_{m} \nabla^{2} C_{m}+\mu_{T} T_{i, j}+\mu_{E} E C_{i, j}-\lambda_{m} C_{m} \\
& \frac{\partial C_{v}}{\partial t}=D_{v} \nabla^{2} C_{v}+\chi T C_{i, j}+\xi C_{f}-\epsilon C_{i, j}-\lambda_{v} C_{v}
\end{aligned}
$$

where $C_{f}, C_{m}, C_{v}$ are the ECM, MDE and VEGF concentration, separately. The $T_{i, j}$ and $\mathrm{EC}_{\mathrm{i}, \mathrm{j}}$ terms represent a tumour cell and an endothelial cell located at a node position $(\mathrm{i}, \mathrm{j})$. Their values are either 1 if a cell is present or 0 if it is not. $D_{m}$ is the MDE diffusion coefficient. $D_{v}$ is VEGF diffusion coefficient. $\delta, \mu_{T}, \mu_{E}, \lambda_{m}, \chi, \xi, \varepsilon, \lambda_{v}$ are positive constants.

To obtain a more realistic oxygen concentration field, the advection and diffusion of oxygen in the vessel network are introduced [27]. The computational space is separated into three domains to characterize three distinct physiological processes, which are (a) the oxygen advection equation inside the vessel, (b) the oxygen flux across the vessel wall and (c) the free oxygen diffusion in the tissue. The detailed equations and methodology of oxygen delivery can be found in Cai et al. [28].

\section{Haemodynamical microenvironment}

The haemodynamic model in this study is based on our previous work on the coupled modelling of intravascular blood flow with interstitial fluid flow [21, 22]. Briefly, the basic equation for the intravascular blood flow is the flux concentration and incompressible flow at each node. Flow resistance is assumed to follow Poiseuille's law in each vessel segment. The interstitial fluid flow is controlled by Darcy's law. The intravascular and interstitial flow is coupled by the transvascular flow, which is described by Starling's law. Blood viscosity is a function of vessel diameter, local haematocrit, and plasma viscosity [29]. In addition, vessel compliance and wall shear stress are correlated to vessel remodelling and vessel collapse (see "Angiogenesis and vessel remodelling" section).

The main equations for blood flow calculation are as follows:

$$
\begin{aligned}
& \mathrm{Q}_{\mathrm{v}}=\frac{\pi \mathrm{R}^{4} \Delta \mathrm{P}_{\mathrm{v}}}{8 \mu \Delta \mathrm{l}} \\
& \mathrm{Q}_{\mathrm{t}}=2 \pi \mathrm{R} \cdot \Delta \mathrm{l} \cdot \mathrm{L}_{\mathrm{p}}\left(\mathrm{P}_{\mathrm{v}}-\mathrm{P}_{\mathrm{i}}-\sigma_{\mathrm{T}}\left(\pi_{\mathrm{v}}-\pi_{\mathrm{i}}\right)\right) \\
& \mathrm{Q}=\mathrm{Q}_{\mathrm{v}}-\mathrm{Q}_{\mathrm{t}}
\end{aligned}
$$


where $\mathrm{Q}$ is the flow rate of each vessel segment, which has a value zero at each node of the vessel network due to the assumption of flux conservation and incompressible flow. $\mathrm{Q}_{\mathrm{v}}$ is the vascular flow rate without fluid leakage; $\mathrm{Q}_{\mathrm{t}}$ is the transvascular flow rate. $\Delta \mathrm{l}$ and $R$ are the mean length and radius of the vessel segment. $P_{v}$ and $P_{i}$ are the intravascular pressure and the interstitial pressure, respectively. Lp is the hydraulic permeability of the vessel wall. $\sigma_{\mathrm{T}}$ is the average osmotic reflection coefficient for plasma proteins; $\pi_{\mathrm{v}}$ and $\pi_{\mathrm{i}}$ are the colloid osmotic pressure of plasma and interstitial fluid, respectively.

The velocity of intravascular $U_{v}$ and interstitial flow $U_{i}$ satisfies

$$
\begin{aligned}
& \mathrm{U}_{\mathrm{v}}=\mathrm{Q} / \pi \mathrm{R}^{2} \\
& \mathrm{U}_{\mathrm{i}}=-\mathrm{K} \nabla \mathrm{P}_{\mathrm{i}} \\
& \nabla \cdot \mathrm{U}_{\mathrm{i}}=\frac{\mathrm{L}_{\mathrm{p}} \mathrm{S}}{\mathrm{V}}\left(\mathrm{P}_{\mathrm{v}}-\mathrm{P}_{\mathrm{i}}-\sigma_{\mathrm{T}}\left(\pi_{\mathrm{v}}-\pi_{\mathrm{i}}\right)\right)
\end{aligned}
$$

where $\mathrm{K}$ is the hydraulic conductivity coefficient of the interstitium; $\mathrm{S} / \mathrm{V}$ is the surface area per unit volume for transport in the interstitium.

\section{Anti-angiogenic therapy}

Endostatin (ES), identified in mice by O'Reilly et al. [30] in 1997, has been considered as one of the most potential anti-angiogenic drugs. It can inhibit endothelial cell proliferation, migration, invasion and tube formation. In the present model, EC apoptosis in response to Endostatin is introduced in the angiogenesis equation [Eq. (1)]

$$
\frac{\partial \mathrm{e}}{\partial \mathrm{t}}=\mathrm{D}_{\mathrm{e}} \nabla^{2} \mathrm{e}-\nabla \cdot\left(\frac{\phi_{\mathrm{c}}}{1+\sigma \mathrm{C}_{\mathrm{v}}} \mathrm{e} \nabla \mathrm{C}_{\mathrm{v}}+\phi_{\mathrm{h}} \mathrm{e} \nabla \mathrm{C}_{\mathrm{f}}\right)+\alpha_{\mathrm{r}}\left(1-\frac{\mathrm{e}}{\mathrm{e}_{0}}\right) \mathrm{e}\left(1-\frac{\epsilon_{\max } C_{\mathrm{ES}}}{\mathrm{C}_{\mathrm{ES}} C_{\mathrm{ES} 50}+\mathrm{C}_{\mathrm{ES}}}\right)
$$

where $\mathrm{e}_{0}$ is the initial EC density. $\varepsilon_{\max }$ is the maximum inhibiting effect of ES on ECs. $\mathrm{C}_{\mathrm{ES} 50}$ is the $\mathrm{ES}$ concentration that induces $50 \%$ of the maximum inhibiting effect. $\mathrm{C}_{\mathrm{ES}}$ is the concentration of ES, which satisfies

$$
\frac{\partial C_{\mathrm{ES}}}{\partial \mathrm{t}}=\nabla \cdot\left(\mathrm{D}_{\mathrm{ES}} \nabla \mathrm{C}_{\mathrm{ES}}\right)+\frac{-\mathrm{R}_{\mathrm{ES}} \mathrm{C}_{\mathrm{ES}}+\mathrm{U}_{\mathrm{I}, \mathrm{ex}}}{\mathrm{V}_{\mathrm{p}}}-\lambda_{\mathrm{ES}} \mathrm{C}_{\mathrm{ES}}
$$

$D_{E S}$ is the diffusion coefficient of ES. $R_{E S}$ is the ES elimination rate in the plasma. $U_{I, e x}$ is the ES injection rate. $V_{p}$ is the volume of the plasma. $\lambda_{E S}$ is the positive coefficient for ES decay of itself.

\section{Simulation setup}

The $3 \mathrm{D}$ model is defined on a $100 \times 100 \times 100$ grid to cover a $1 \mathrm{~mm} \times 1 \mathrm{~mm} \times 1 \mathrm{~mm}$ volume, so the grid length corresponds approximately to the size of a tumour cell, i.e. $10 \mu \mathrm{m}$. Two separate lattices are constructed for tumour cells (TCs) and endothelial cells (ECs), with the constraint that each grid can contain only one single cell at each time point. We assume that there are two main parallel arterioles located at plane $y=100$, which branch to form capillaries as initial pre-existing vasculature of the model (Fig. 2). 


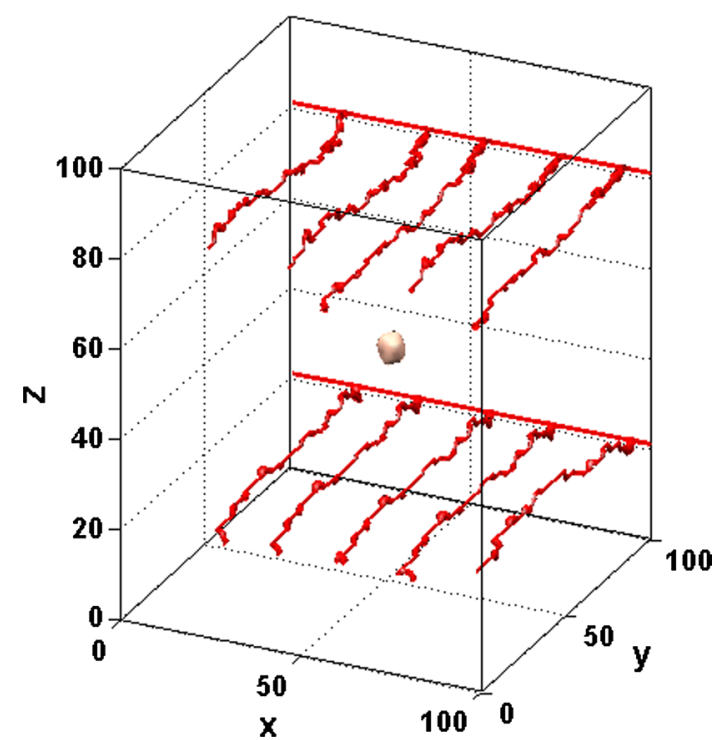

Fig. 2 The initial pre-existing vasculature and tumour morphology of the model

Dirichlet boundary conditions of the chemicals are used in the simulation field. The initial condition of ECM density is set to be 1and other chemicals' concentration (oxygen, VEGF and MDEs) are 0 . The chemicals' concentrations are solved to steady state at each time step of the simulation with an inner iteration step of $5 \mathrm{~s}$. The oxygen concentration has been normalized to be $0-1$ in the "Results" section.

The total difference of $P_{v}$ through the two main parallel arterioles from plane $x=100$ to $\mathrm{x}=0$ is set to be $3.5 \mathrm{mmHg}$ as the driving force of blood in the network (or the boundary condition). The distribution of red blood cells (RBCs) at a microvascular bifurcation is calculated based on the blood rheology simulation (see Wu et al. [21]).

Initially, 20 proliferating cells are placed in the central computational area (see the tumour morphology in Fig. 2). As the tumour grew with time, the metabolic microenvironment such as oxygen, VEGF and other chemicals concentrations are calculated accordingly. At the same time, tumour vessels undergo remodelling and collapse in response to both chemical environment and haemodynamical environment. As a consequence, the local oxygen supply changes with the updated tumour vasculature, which determines the different phenotypes of tumour cells in next time step. The initial concentration of anti-angiogenic drug (ES) is $\mathrm{e}_{0}=2.0 \times 10^{-9} \mathrm{~mol} \mathrm{~L}^{-1}$, and assumed of having an injection dose of $\mathrm{ES}^{*}=20 \mathrm{mg} \mathrm{kg}^{-1}$ day $^{-1}$ after different time point of tumour growth $\left(\mathrm{T}_{\mathrm{inj}}=50, \mathrm{~T}_{\mathrm{inj}}=100\right.$ and $\left.\mathrm{T}_{\mathrm{inj}}=150\right)$. All simulations were performed on an Intel Xeon 3.2 GHz CPU, 24 GB Memory desktop. The parameter values of baseline model are listed in Table 2.

\section{Results}

Tumour growth and angiogenesis without anti-angiogenic drug

Figure 3 shows the history of tumour growth and angiogenesis without anti-angiogenic drug. Tumour vessels are represented by red tubes. At the early stage, tumour cells 
Table 2 Parameter values used in the simulation

\begin{tabular}{|c|c|c|c|}
\hline Parameter & Value & Description & Reference \\
\hline$\Delta \mathrm{l}$ & $10 \mu \mathrm{m}$ & Lattice constant & \\
\hline $\mathrm{R}_{0}$ & $4 \mu \mathrm{m}$ & Origin radius of the capillary & \\
\hline$D_{e}$ & $10^{-9} \mathrm{~cm}^{-3} \mathrm{~s}^{-1}$ & EC diffusion coefficient & {$[20]$} \\
\hline$\phi_{c}$ & $2.6 \times 10^{3} \mathrm{~cm}^{-3} \mathrm{M}^{-1} \mathrm{~s}^{-1}$ & EC chemotaxis coefficient & [20] \\
\hline$\phi_{\mathrm{h}}$ & $10^{3} \mathrm{~cm}^{-3} \mathrm{M}^{-1} \mathrm{~s}^{-1}$ & EC haptotaxis coefficient & {$[20]$} \\
\hline$L_{p}^{T}$ & $2.8 \times 10^{-7} \mathrm{~cm}\left(\mathrm{mmHg} \mathrm{s}^{-1}\right.$ & Vessel permeability in tumour tissue & {$[31]$} \\
\hline$L_{p}^{N}$ & $0.36 \times 10^{-7} \mathrm{~cm}(\mathrm{mmHg} \mathrm{s})^{-1}$ & Vessel permeability in normal tissue & {$[31]$} \\
\hline$P_{c}$ & $3 \mathrm{mmHg}$ & Vessel collapse pressure & {$[26]$} \\
\hline E & $6.5 \mathrm{mmHg}$ & Vessel compliance coefficient & {$[26]$} \\
\hline$b$ & 0.1 & Vessel compliance index & {$[26]$} \\
\hline$D_{m}$ & $10^{-9} \mathrm{~cm}^{-3} \mathrm{~s}^{-1}$ & MDE diffusion coefficient & {$[24]$} \\
\hline$\delta$ & $1.3 \times 10^{2} \mathrm{~cm}^{-3} \mathrm{M}^{-1} \mathrm{~s}^{-1}$ & ECM degradation coefficient & {$[23]$} \\
\hline$\mu_{T}$ & $1.7 \times 10^{-18}$ Mcells $^{-1} \mathrm{~s}^{-1}$ & MDE production by TC & {$[23]$} \\
\hline$\mu_{\mathrm{E}}$ & $0.3 \times 10^{-18}$ Mcells $^{-1} \mathrm{~s}^{-1}$ & MDE production by EC & {$[23]$} \\
\hline$\lambda$ & $1.7 \times 10^{-8} s^{-1}$ & MDE decay coefficient & {$[24]$} \\
\hline$D_{v}$ & $2.9 \times 10^{-7} \mathrm{~cm}^{-3} \mathrm{~s}^{-1}$ & VEGF diffusion coefficient & {$[20]$} \\
\hline$x$ & $10^{-17}$ Mcells $^{-1} \mathrm{~s}^{-1}$ & VEGF production by TC & {$[32]$} \\
\hline$\xi$ & $10^{-3} \mathrm{~cm}^{-3} \mathrm{~s}^{-1}$ & VEGF production in ECM & {$[23]$} \\
\hline$\varepsilon$ & $10^{-20}$ Mcells $^{-1} \mathrm{~s}^{-1}$ & VEGF consumption by EC & {$[32]$} \\
\hline$\theta$ & $10^{-8} s^{-1}$ & VEGF decay coefficient & {$[32]$} \\
\hline$e_{0}$ & $2.0 \times 10^{-9} \mathrm{~mol} \mathrm{~L}^{-1}$ & Initial EC density & [33] \\
\hline$\varepsilon_{\max }$ & 1 & Max inhibiting effect of ES on ECs & {$[33]$} \\
\hline$C_{E S 50}$ & $2.288 \times 10^{-8} \mathrm{~mol} \mathrm{~L}^{-1}$ & $\begin{array}{l}\text { ES concentration that induces } 50 \% \text { of the maximum } \\
\text { inhibiting effect }\end{array}$ & [33] \\
\hline$D_{E S}$ & $2.9 \times 10^{-7} \mathrm{~cm}^{-3} \mathrm{~s}^{-1}$ & Diffusion coefficient of ES & {$[33]$} \\
\hline$R_{E S}$ & $5.54 \times 10^{-5} \mathrm{Ls}^{-1}$ & ES elimination rate in the plasma & [33] \\
\hline$U_{l, e x}$ & $20 \mathrm{mg}(\mathrm{kg} \times \text { day })^{-1}$ & ES injection rate & [33] \\
\hline$V_{p}$ & $10^{-3} \mathrm{~L}$ & Volume of the plasma & {$[33]$} \\
\hline$\lambda_{E S}$ & $10^{-8 / 5}$ & ES decay coefficient & Estimated \\
\hline
\end{tabular}

rapidly uptake oxygen diffused from pre-existing vessels, which is known as avascular stage of tumour growth $(\mathrm{T}=50)$. The angiogenesis phase is triggered on due to the drop of oxygen levels. Well circulated angiogenic vessel networks can be seen at $\mathrm{T}=100$, which suggests that the neo-vasculature generates a new supply of oxygen for further tumour growth. In addition, vascularized tumours are more likely to develop dendritic structures compared to round tumours in avascular phase. This indicates that tumour angiogenesis may reinforce the heterogeneity of tumour microenvironment, favoring a more aggressive morphology of solid tumours $(\mathrm{T}=200)$.

The numbers of tumour cells with different phenotypes at different time step are presented as curves in Fig. 4. The number of total tumour cells and proliferating cells increase continually with time in the simulation. However, the number of quiescent cells have a slight reduction in the period of $T=70$ to $T=100$. At the same time, necrotic cells remain linear expansion, due to the inadequate oxygen supply by pre-existing vessel network. As a consequence, angiogenesis phase occurs at this time (see Fig. 3). The proliferating cell population shows exponential increase after the emergence of angiogenic 


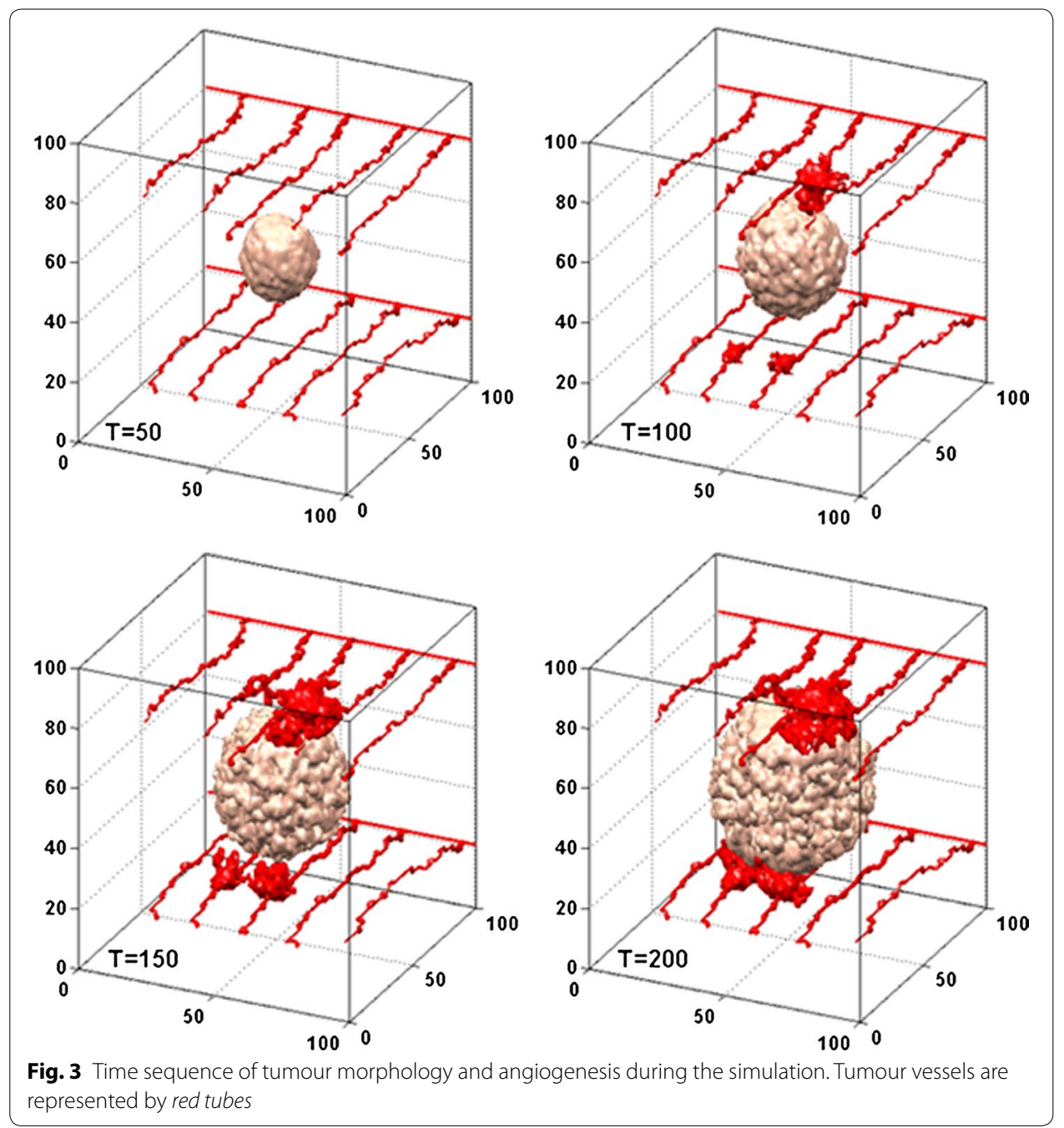

tumour vessels, which demonstrates that the feedback mechanisms of the coupled model work well in the simulation.

\section{Tumour growth and angiogenesis with anti-angiogenic drug}

In order to study the influence of anti-angiogenic drug ES on tumour growth and morphology, we tested five drug strategies with different drug concentration and different injection time. Compared to base case (Fig. 5a), a low ES concentration is found to induce little growth suppression (Fig. 5b), while large doses of ES (ES $=10 \mathrm{ES} *$ ) can produce significant tumour cell apoptosis (Fig. 5c). The effect of different injection time of ES on tumour growth is shown in Fig. $5 \mathrm{~d}-\mathrm{f}$. It is noteworthy that a drastically suppression of tumour size is generated when ES is inserted at simulation time 100, which is concomitant with the emergence of angiogenesis phase. As shown by growth history curves in Fig. 6, up to $50 \%$ of reduction of total tumour cells number can be seen after ES treatment on $\mathrm{T}_{\mathrm{inj}}=100$. However, only 30 and $21 \%$ of reduction can achieved respectively if injection time of ES is earlier or later than simulation time 100. 


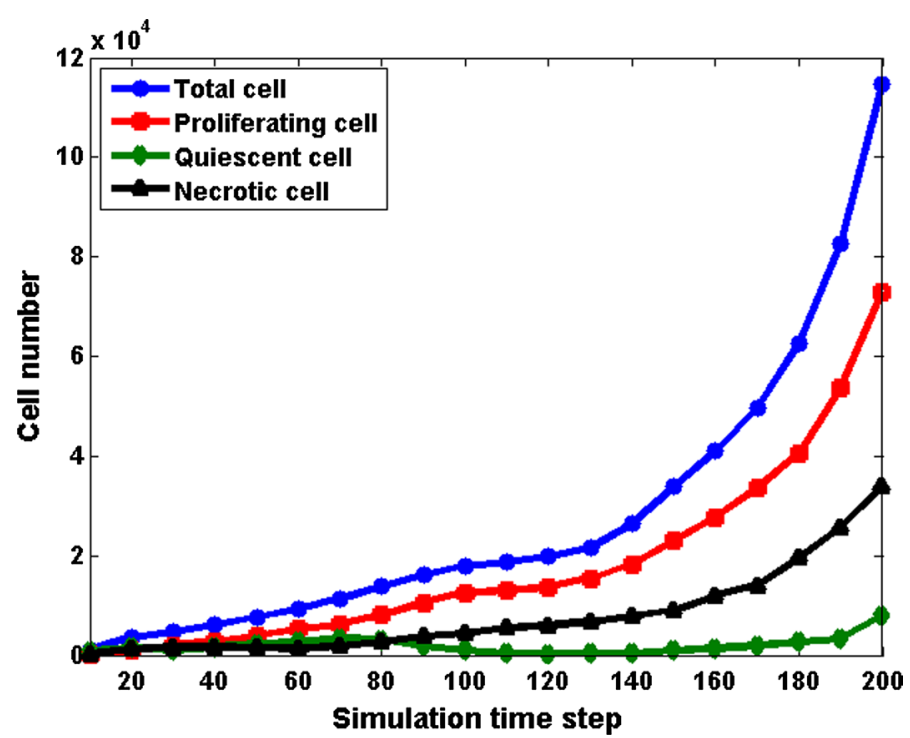

Fig. 4 Growth history of tumour cells with different phenotypes. Blue total tumour cells; red proliferating cells; green quiescent cells; black necrotic cells
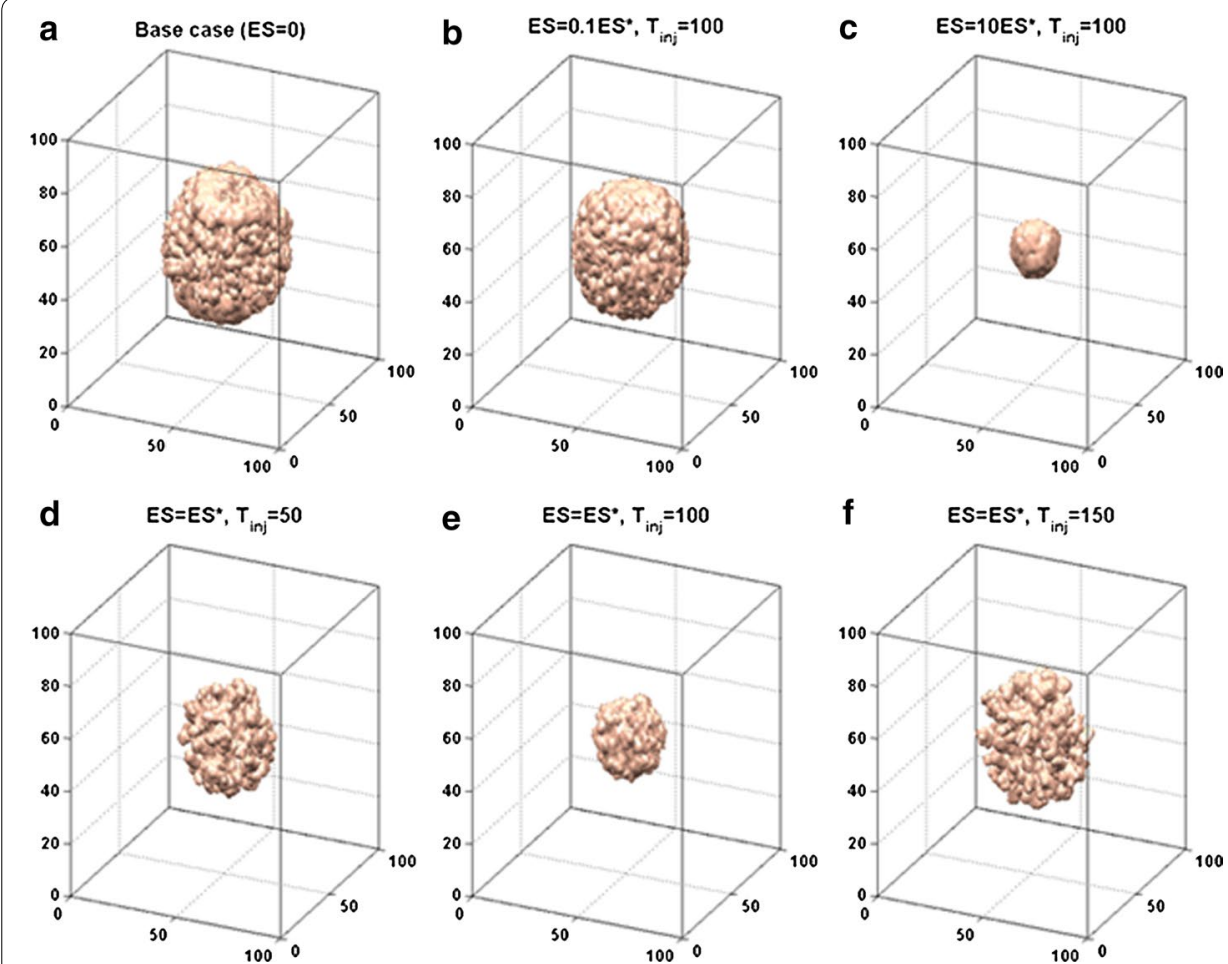

Fig. 5 Tumour morphology at $T=200$ following different anti-angiogenic drug strategies. a Base case with no anti-angiogenic drug, as described in "Tumour growth and angiogenesis without anti-angiogenic drug" section. b, c The anti-angiogenic drug ES is inserted at $\mathrm{T}_{\mathrm{inj}}=100$, with different drug concentrations $\left(E S=0.1 E S^{*}, E S=10 E S^{*}\right)$. d, e, $\mathbf{f}$ The effect of ES on tumour growth with same dose but different injection time, $T_{\text {inj }}=50,100,150$ respectively 


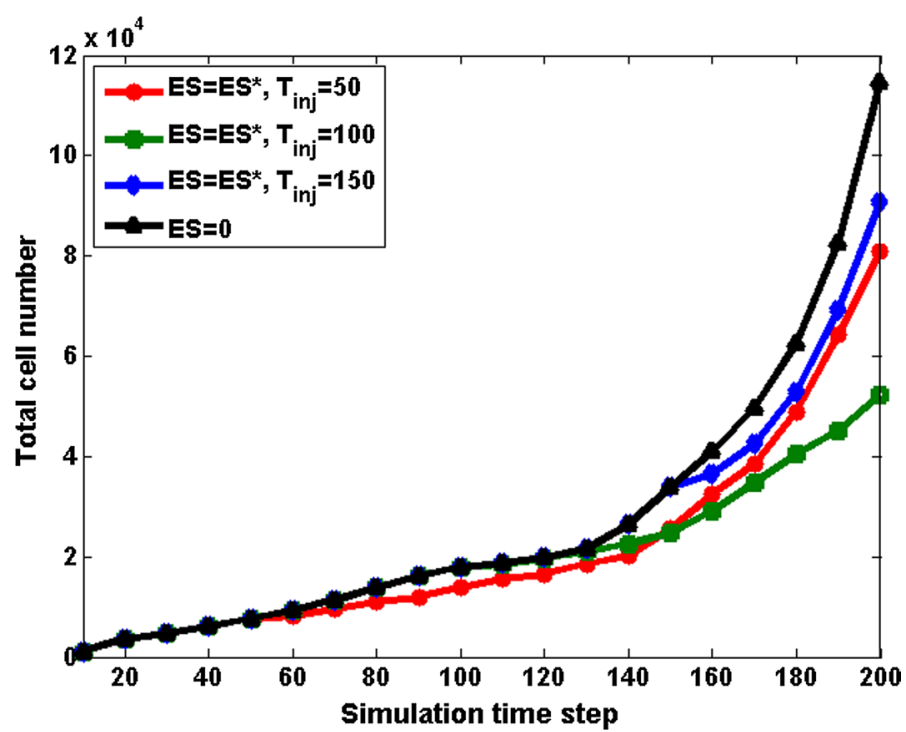

Fig. 6 Growth history curves of total tumour cells following different anti-angiogenic drug strategies

\section{Metabolical and haemodynamical microenvironment}

The model predicts the chemical and haemodynamical substances distribution including oxygen, VEGF, interstitial pressure and vessel radius, as shown in Fig. 7. The tumour centre is always in a hypoxic state, while high concentration of oxygen occurred near
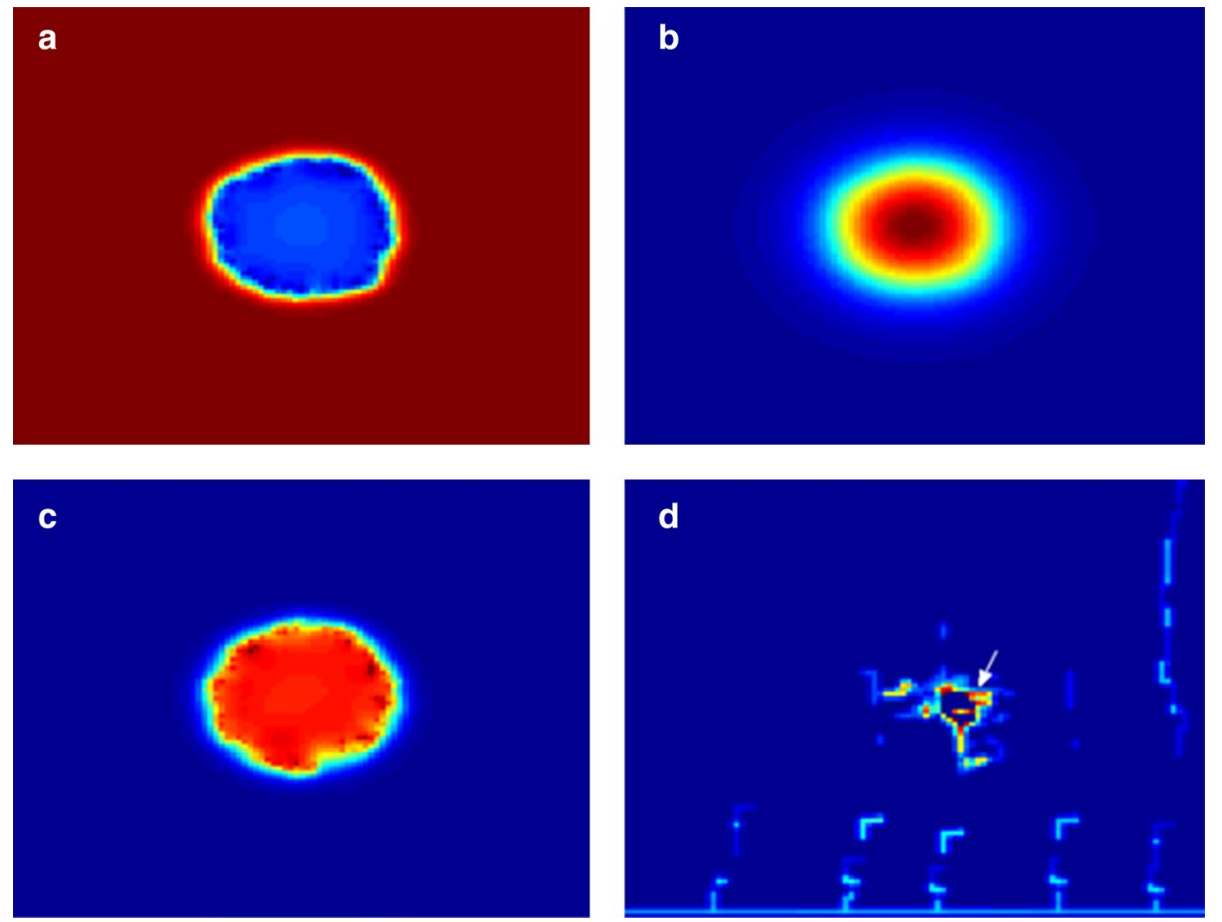

Fig. 7 Chemical and haemodynamical microenvironment on $T=200$, at plane $z=20$. a Oxygen distribution. $\mathbf{b}$ VEGF distribution. $\mathbf{c}$ Interstitial pressure. $\mathbf{d}$ Vessel radius distribution. Arrow indicates the vessel dilation. Intensity: red (high), blue (low) 
the tumour edge due to the localized angiogenic vasculature at the tumour periphery (Fig. 7a). VEGF has a high density inside the tumour associated with hypoxic tumour cells, attracting neo-vessels growth towards the tumour (Fig. 7b). Interstitial pressure $P_{i}$ is significantly higher than that of surrounding tissue and drops dramatically in the tumour margin (Fig. 7c), which compromises the delivery of therapeutic agents both across the blood vessel wall and interstitium in tumours. Vessel radius shows unevenly distributed, with few dilated functional vessels left in the tumour centre (Fig. 7d, arrow). The large variation in vessel diameters will increase the flow resistance in tumours microvasculature, which has been observed in published experiments [5].

\section{Parameter sensitivity}

In the current model, the main factors that directly influence ECs distribution are the chemotaxis of VEGF, the haptotaxis of ECM, the vessel remodelling in response to local haemodynamical microenvironment and the inhibition effect due to ES treatment. The sensitivity of tumour growth to the important parameters are analyzed, including chemotaxis coefficient $\phi_{c}$, haptotaxis coefficient $\phi_{h}$, wall permeability of tumour capillaries $L_{p}$ and maximum inhibiting effect of ES $\varepsilon_{\max }$. The variation ranges of tested parameters are \pm 1 and $\pm 10 \%$ from their default values listed in Table 2. As shown in Fig. 8, the number of total tumour cells is quite robust with respect to varied parameters (less than 15\% changes). The most sensitive parameter to this model is the maximum inhibiting effect of ES $\varepsilon_{\max }$.

\section{Discussion}

In this paper, we proposed a multi-scale coupled modelling system to investigate the dynamic process of tumour growth and angiogenesis in response to the changes in chemical and haemodynamical microenvironment caused by anti-angiogenic therapy. At tissue level, we considered tumour growth, tumour angiogenesis and vessel remodelling.

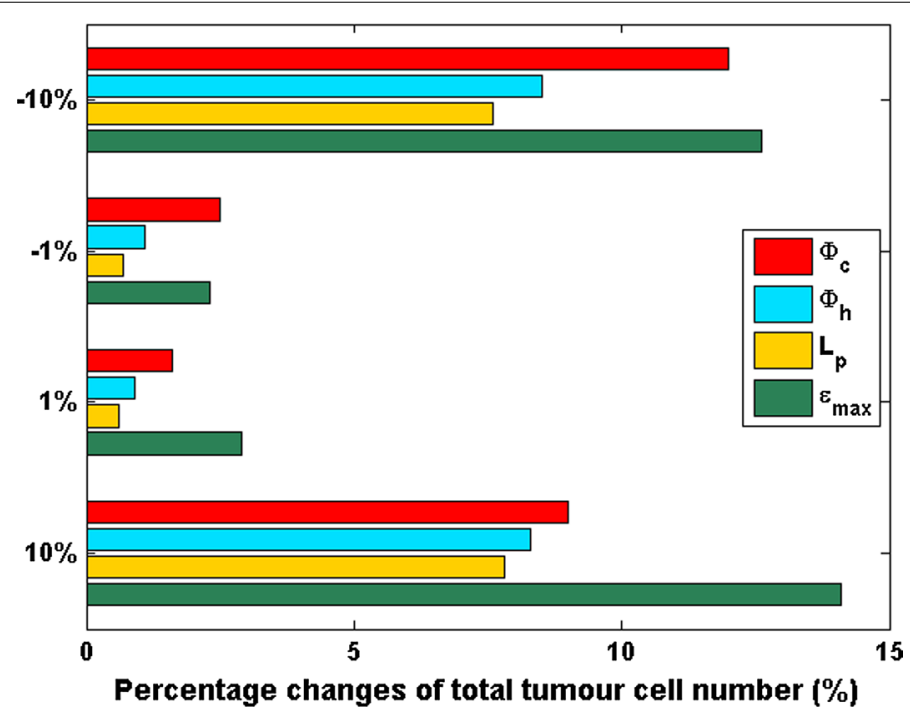

Fig. 8 Percentage changes of number of total tumour cells with respect to varied parameters 
Tumour growth and neo-vessel growth are coupled by the chemical microenvironment including oxygen, VEGF, ECM and MDE, which are described by continuous partial differential equations. At the same time, haemodynamic calculation is carried out by coupling intravascular blood flow and interstitial fluid flow. According to the microenvironmental information, detailed and comprehensive mechanisms of tumour cell phenotype and endothelial cell proliferation and migration are generated at cellular level, which in turn influence the modelling at intratumoral and tissue levels. In addition, we extended the model to study the dynamic changes of tumour growth in response to antiangiogenic therapy. Anti-angiogenic drug ES is assumed to target immature vessels and may cause endothelial cell apoptosis.

Simulation results revealed multiple characteristics of tumour progression, including tumour expansion, morphology changes and angiogenesis. The growth curves of cell population with different cell phenotypes are consistent with the pathophysiological knowledge. Furthermore, different anti-angiogenic drug strategies are designed to test the influence of ES on tumour growth and morphology. The largest reduction of tumour size is found when ES is injected at simulation time 100, which is concomitant with the emergence of angiogenesis phase. With pharmacokinetics information of specific anti-angiogenic drugs, the proposed model can be adopted for cancer drug discovery and testing.

There are a few major limitations of the present work. On calculating the tumour mechanical environment, only interstitial fluid static pressure was included. Mechanical stress caused by the rapid proliferation of tumour cells was not simulated in the model. This solid mechanical stress may influence tumour cell behaviour. In addition, although most of the simulation parameters were set based on published experimental data, some of them cannot be found, such as tumour migration speed.

\section{Conclusions}

A multi-scale coupled modelling system to investigate the dynamic process of tumour growth and angiogenesis in response to the changes in chemical and haemodynamical microenvironment during the anti-angiogenic therapy, is established. The simulation results demonstrate the inhibition of tumour growth and progression due to the reduction of neo-vasculature in response to anti-angiogenic therapy. Different anti-angiogenic drug strategies can be designed based on the proposed mathematical model, to test the influence of anti-angiogenic drug on tumour growth and morphology, with pharmacokinetics information of specific drugs

Declarations

Authors' contributions

YC and ZL were responsible for the design and computational modeling. YC and JZ were responsible for data analysis part. All authors read and approved the final manuscript.

Author details

${ }^{1}$ State Key Laboratory of Bioelectronics, Southeast University, Nanjing, China. ${ }^{2}$ School of Biological Sciences and Medical Engineering, Southeast University, 2 Sipailou, Nanjing 200018, China. ${ }^{3}$ Shanghai Pulmonary Hospital, Tongji University, Shanghai, China.

\section{Acknowledgements}

This research is supported by the National Basic Research Program of China (973 Program) (No. 2013CB733800), the National Nature Science Foundation of China (No. 11302050, No. 11272091), the Nature Science Foundation of Jiangsu Province (No. BK20130593).

Competing interests

The authors declare that they have no competing interests. 


\section{About this supplement}

This article has been published as part of BioMedical Engineering OnLine Volume 15 Supplement 2, 2016. Computational and experimental methods for biological research: cardiovascular diseases and beyond. The full contents of the supplement are available online http://biomedical-engineering-online.biomedcentral.com/articles/supplements/ volume-15-supplement-2.

Availability of data and materials

All relevant data are within the paper.

\section{Funding}

Publication charges for this article have been funded by the National Nature Science Foundation of China (No. 11302050)

\section{Published: 28 December 2016}

\section{References}

1. Folkman J, Bach M, Rowe JW, et al. Tumor angiogenesis-therapeutic implications. N Engl J Med. 1971;285:1182-6.

2. Ferrara N, Gerber HP, LeCouter J. The biology of VEGF and its receptors. Nat Med. 2003;9(6):669-76.

3. Potente M, Gerhardt H, Carmeliet P. Basic and therapeutic aspects of angiogenesis. Cell. 2011;146:873-87.

4. Vasudev NS, Reynolds AR. Anti-angiogenic therapy for cancer: current progress, unresolved questions and future directions. Angiogenesis. 2014;17:471-94.

5. Fukumura D, Jain RK. Tumor microvasculature and microenvironment: targets for anti-angiogenesis and normalization. Microvasc Res. 2007:74:72-84

6. Ellis LM, Hicklin DJ. VEGF-targeted therapy: mechanisms of anti-tumour activity. Nat Rev Cancer. 2008;8(8):579-91.

7. Gialeli $C$, et al. Roles of matrix metalloproteinases in cancer progression and their pharmacological targeting. FEBS J. 2011;278:16-27.

8. Ribatii D. Endogenous inhibitors of angiogenesis: a historical review. Leuk Res. 2009;33:638-44.

9. Zulato E, Curtarello M, Nardo G, et al. Metabolic effects of anti-angiogenic therapy in tumors. Biochimie. 2012;94:925-31.

10. Lambrechts D, et al. Markers of response for the antiangiogenic agent bevacizumab. J Clin Oncol. 2013;31:1219-30.

11. Roodink I, Leenders WPJ. Targeted therapies of cancer: angiogenesis inhibition seems not enough. Cancer Lett. 2010;299:1-10.

12. Jain RK. Normalizing tumor microenvironment to treat cancer: bench to bedside to biomarkers. J Clin Oncol. 2013:31:2205-18.

13. Harris AL. Hypoxia: a key regulatory factor in tumour growth. Nat Rev Cancer. 2002;2:38-47.

14. Jain RK. Normalization of tumor vasculature: an emerging concept in antiangiogenic therapy. Science. 2005;307:58-62.

15. Billy F, et al. A pharmacologically based multiscale mathematical model of angiogenesis and its use in investigating the efficacy of a new cancer treatment strategy. JTheor Biol. 2009;260:545-62.

16. Plank MJ, Sleeman BD. A reinforced random walk model of tumour angiogenesis and anti-angiogenic strategies. Math Med Biol. 2003:20:135-81.

17. Jain RK, et al. Effect of vascular normalization by antiangiogenic therapy on interstitial hypertension, peritumor edema, and lymphatic metastasis: insights from a mathematical model. Cancer Res. 2007;67:2729-35.

18. Phipps C, Kohandel M. Mathematical model of the effect of interstitial fluid pressure on angiogenic behavior in solid tumors. Comput Math Methods Med. 2011:2011:843765.

19. Alarcón T, Page KM. Mathematical models of the VEGF receptor and its role in cancer therapy. J R Soc Interface. 2007:4:283-304.

20. Anderson ARA, Chaplain MAJ. Continuous and discrete mathematical models of tumor-induced angiogenesis. Bull Math Biol. 1998;60:857-900.

21. Wu J, Xu SX, Long Q, et al. Coupled modeling of blood perfusion in intravascular, interstitial spaced in tumor microvasculature. J Biomech. 2008;41:996-1004.

22. Wu J, Long Q, Xu SX, et al. Study of tumor blood perfusion and its variation due to vascular normalization by antiangiogenic therapy based on 3D angiogenic microvasculature. J Biomech. 2009;42:712-21.

23. Cai Y, Xu SX, Wu J, et al. Coupled modelling of tumour angiogenesis, tumour growth, and blood perfusion. J Theor Biol. 2011;279:90-101.

24. Cai Y, Wu J, Li ZY, et al. Mathematical modelling of a brain tumour initiation and early development: a coupled model of glioblastoma growth, pre-existing vessel co-option, angiogenesis and blood perfusion. PLoS ONE. 2016;11(3):e0150296.

25. Welter $\mathrm{M}$, Bartha $\mathrm{K}$, Rieger $\mathrm{H}$. Vascular remodelling of an arterio-venous blood vessel network during solid tumour growth. J Theor Biol. 2009;259:405-22.

26. Netti PA, Roberge S, Boucher Y, et al. Effect of transvascular fluid exchange on pressure-flow relationship in tumors: a proposed mechanism for tumor blood flow heterogeneity. Microvasc Res. 1996;52:27-46.

27. Fang $Q$, Sakadžić $S$, Ruvinskaya $L$, et al. Oxygen advection and diffusion in a three dimensional vascular anatomical network. Opt Express. 2008;16:17530-41

28. Cai Y, Zhang J, Wu J, et al. Oxygen transport in a three-dimensional microvascular network incorporated with early tumour growth and preexisting vessel cooption: numerical simulation study. Biomed Res Int. 2015;2015:476964. 
29. Pries AR, Secomb TW. Microvascular blood viscosity in vivo and the endothelial surface layer. Am J Physiol Heart Circ Physiol. 2005;289:H2657-64.

30. O'Reilly MS, et al. Endostatin: an endogenous inhibitor of angiogenesis and tumor growth. Cell. 1997;88(2):277-85

31. Baxter LT, Jain RK. Transport of fluid and macromolecules in tumors. I. Role of interstitial pressure and convection. Microvasc Res. 1989;37:77-104.

32. Alarcón T, Owen MR, Byrne HM, et al. Multiscale modelling of tumour growth and therapy: the influence of vessel normalisation on chemotherapy. Comput Math Methods Med. 2006;7(2-3):85-119.

33. Cai Y, Wu J, Xu SX, et al. Numerical simulation of inhibiting effects on solid tumour cells in anti-angiogenic therapy: application of coupled mathematical model of angiogenesis with tumour growth. Appl Math Mech. 2011;32(10):1287-96.

Submit your next manuscript to BioMed Central and we will help you at every step:

- We accept pre-submission inquiries

- Our selector tool helps you to find the most relevant journal

- We provide round the clock customer support

- Convenient online submission

- Thorough peer review

- Inclusion in PubMed and all major indexing services

- Maximum visibility for your research

Submit your manuscript at www.biomedcentral com/submit 